

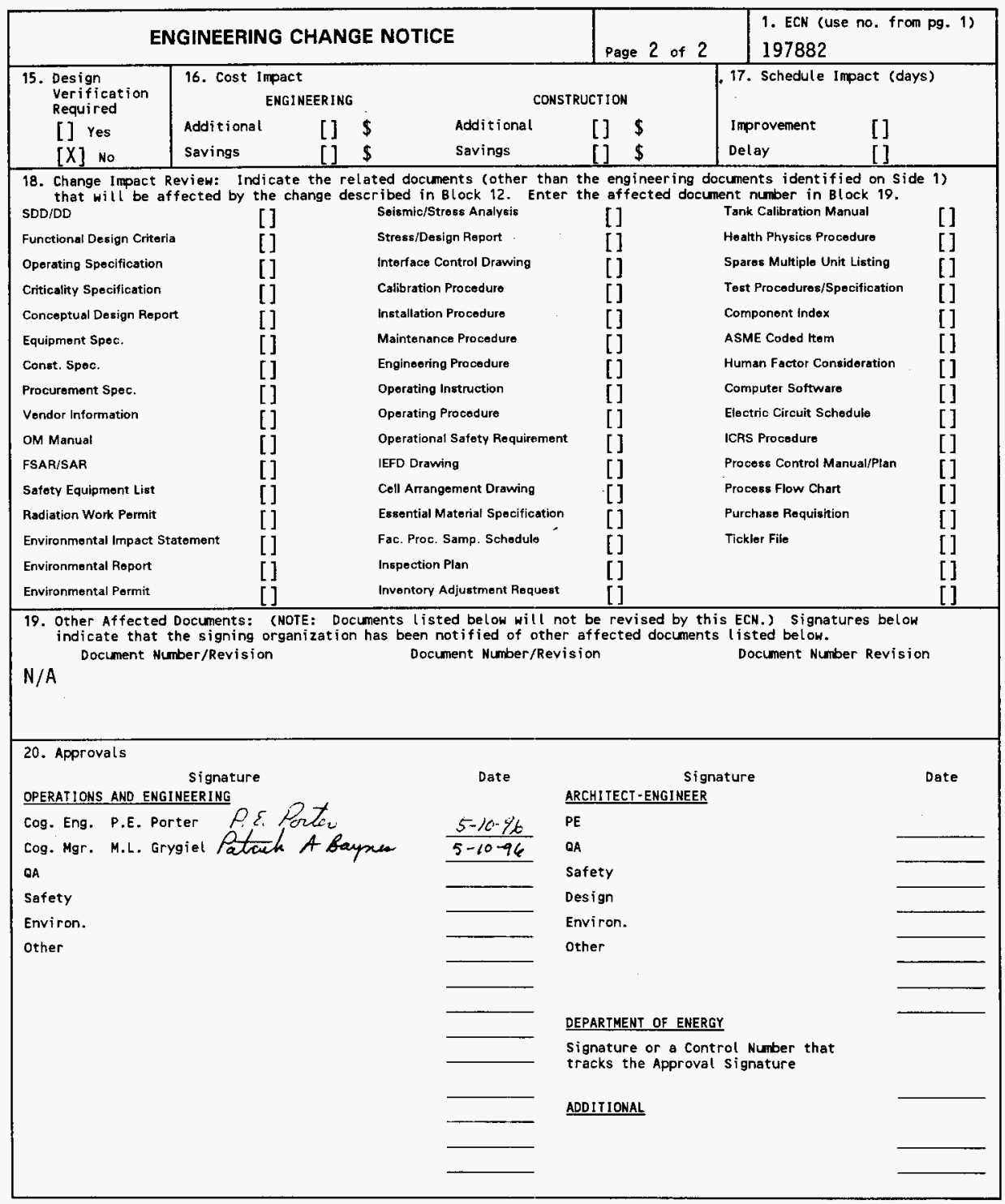




\title{
Hanford Site Technical Baseline Database
}

\author{
P.E. Porter \\ WHC, Richland, WA 99352 \\ U.S. Department of Energy Contract DE-AC06-87RL10930

$\begin{array}{lll}\text { EDT/ECN: } & 197882 & \text { UC: } 905 \\ \text { Org Code: } & 02100 & \text { Charge Code: } \\ \text { B\&R Code: } & \text { EW3120075 } & \text { Total Pages: } 14\end{array}$

Key Words: Hanford, Site, Technical, Baseline, Database, RDD-100, Systems Engineering

Abstract: This document includes a cassette tape that contains the Hanford specific files that make up the Hanford Site Technical Baseline Database as of May 10, 1996. The cassette tape also includes the delta files that dellinate the differences between this revision and revision 3 (April 10, 1996) of the Hanford Site Technical Baseline Database.

TRADEMARK DISCLAIMER. Reference herein to any specific comercial product, process, or service by trade name, trademark, manufacturer, or otherwise, does not necessarily constitute or imply its endorsement, recommendation, or favoring by the United States Government or any agency thereof or its contractors or subcontractors.

Printed in the United States of America. To obtain copies of this document, contact: WHC/BCS Document Control Services, P.O. Box 1970, Mailstop H6-08, Richland WA 99352, Phone (509) 372-2420; Fax (509) 376-4989.
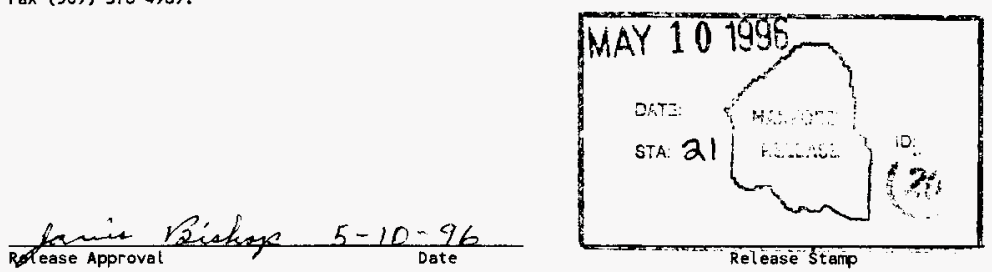

\section{Approved for Public Release}




\begin{tabular}{|c|c|c|c|c|}
\hline & RECORD OF REVISION & \multicolumn{2}{|c|}{$\begin{array}{l}\text { (1) Document Number } \\
\text { WHC-SD-GN-CSWD- } \\
20012\end{array}$} & Page $i$ \\
\hline \multicolumn{5}{|c|}{$\begin{array}{l}\text { (2) Title } \\
\text { Hanford Site Technical Baseline Database }\end{array}$} \\
\hline \\
\hline \multirow{2}{*}{ (3) Revision } & \multirow{2}{*}{$\begin{array}{l}\text { CHANGE CONTROL RECORD } \\
\text { (4) Description of Change - Replace, Add, and Delete Pages }\end{array}$} & \multicolumn{3}{|c|}{ Authorized for Release } \\
\hline & & (5) Cog. Engr. & (6) & Mgr. Date \\
\hline 0 & (7) Initial Release, EDT-603662 & N/A & $\begin{array}{l}N / A \\
10 / 0\end{array}$ & \\
\hline 1 & $\begin{array}{l}\text { Updated Hanford Site Technical Baseline } \\
\text { Database to incorporate information from } \\
\text { continued development activities. Changes } \\
\text { made at the site-level and within functions } \\
4.2 \text { and } 4.4 \text {. See ECN } 614747\end{array}$ & P. Porter & $\begin{array}{l}\text { J. } 0 \\
01 / 2\end{array}$ & $\begin{array}{ll}1 \text { ings } \\
95\end{array}$ \\
\hline 2 & $\begin{array}{l}\text { Updated Hanford Site Technical Baseline } \\
\text { Database. Changes made at the site-level } \\
\text { and within Functions } 4.1,4.2,4.6,4.7, \\
5.0 \text {. See ECN } 197879\end{array}$ & P. Porter & $\begin{array}{l}\text { M. } G \\
08 / 1\end{array}$ & giel \\
\hline 3 & $\begin{array}{l}\text { Updated Hanford Site Technical Baseline } \\
\text { Database. Changes made at the sitelevel } \\
\text { and within all Functions. See ECN } 197881 \\
\end{array}$ & P. Porter & $\begin{array}{l}M . \\
04 / 1\end{array}$ & $\begin{array}{l}\text { giel } \\
96\end{array}$ \\
\hline 4 FS & $\begin{array}{l}\text { Updated Hanford Site Technical Baseline } \\
\text { Database. Changes made at the Site-Level } \\
\text { and within all Function. See ECK } 197882\end{array}$ & $\begin{array}{l}\text { P. Porter } \\
\text { EPutiv }\end{array}$ & $\begin{array}{l}\text { M. } \\
\text { og/t }\end{array}$ & $\begin{array}{l}\text { giel } \\
96 \\
\text { A Sayas }\end{array}$ \\
\hline & & & & \\
\hline & & & & \\
\hline & & & & \\
\hline & & & & \\
\hline & & & & \\
\hline & & & & \\
\hline & & & & \\
\hline & & & & \\
\hline & & & & \\
\hline & & & & \\
\hline & & & & \\
\hline & & & & \\
\hline & & & & \\
\hline & & & & \\
\hline & & & & \\
\hline & & & & \\
\hline & & & & \\
\hline & & & & \\
\hline & & & & \\
\hline
\end{tabular}




\subsection{PURPOSE AND SCOPE}

The attached cassette tape contains the Hanford specific files (Table 1) that make up the Hanford Site Technical Baseline Database. The cassette tape also includes the delta files (Table 2) that delineate the differences between this revision and revision 0 of the Hanford Site Technical Baseline Database. This information is being managed and maintained on the Hanford RDD-100 System, which uses the capabilities of RDD-100, a systems engineering software system of Ascent Logic Corporation (ALC). This revision of the Hanford Site Technical Baseline Database uses RDD-100 version 4.0.3 (see Table 3). Directories reflect those controlled by the Hanford RDD-100 System Administrator. Table 4 provides information regarding the platform.

\subsection{RESPONSIBILITIES}

The Systems Engineering Integration organization is responsible for the development and maintenance of this document.

*This document has been extensively revised; therefore, no revision bars are used to indicate changes. 
WESTINGHOUSE HANFORD COMPANY

Hanford Site Technical Baseline Database
WHC-SD-GN-CSWD-20012

Rev. 4

Page:

2 of 13

Effective Date:

\begin{tabular}{|c|c|c|c|}
\hline & File Size & Date & Time \\
\hline \multicolumn{4}{|c|}{$\begin{array}{l}\text { Directory: cmbl/site/New/Site } \\
\text { \{Contains Hanford Site Technical Baseline site-level data.\} }\end{array}$} \\
\hline Files: $\begin{aligned} \text { HSTB-Rev4-Empty.im } \\
\text { HSTB-Rev4.im } \\
\text { HSTB-Rev4.rdt }\end{aligned}$ & $\begin{array}{l}7774528 \\
22313756 \\
11996295\end{array}$ & $\begin{array}{l}\text { May } 101996 \\
\text { May } 101996 \\
\text { May } 101996\end{array}$ & $\begin{array}{l}10: 36 \\
10: 37 \\
10: 37\end{array}$ \\
\hline \multicolumn{4}{|l|}{$\begin{array}{l}\text { Directory: cmbl/site/New/Reports } \\
\text { \{Contains WHC developed applications.\} }\end{array}$} \\
\hline Files: SystemSegmentSpecification.rpt & 507869 & May 101996 & $10: 38$ \\
\hline \multicolumn{4}{|l|}{$\begin{array}{l}\text { Directory: } \mathrm{cmbl} / \mathrm{site} / \mathrm{New} / \mathrm{Sch} \text { ema } \\
\text { \{Contains schema.\} }\end{array}$} \\
\hline File: HSTB-Rev4-Schema.sct & 118117 & May 101996 & $05: 58$ \\
\hline \multicolumn{4}{|l|}{ Directory: cmbl/site/New/Site/Documents } \\
\hline File: HanfordSiteCleanupSpecification.ps & 583088 & May 101996 & $10: 38$ \\
\hline
\end{tabular}


Table 2. Hanford Site Technical Baseline Database Hanford Specific Files - Delta Files

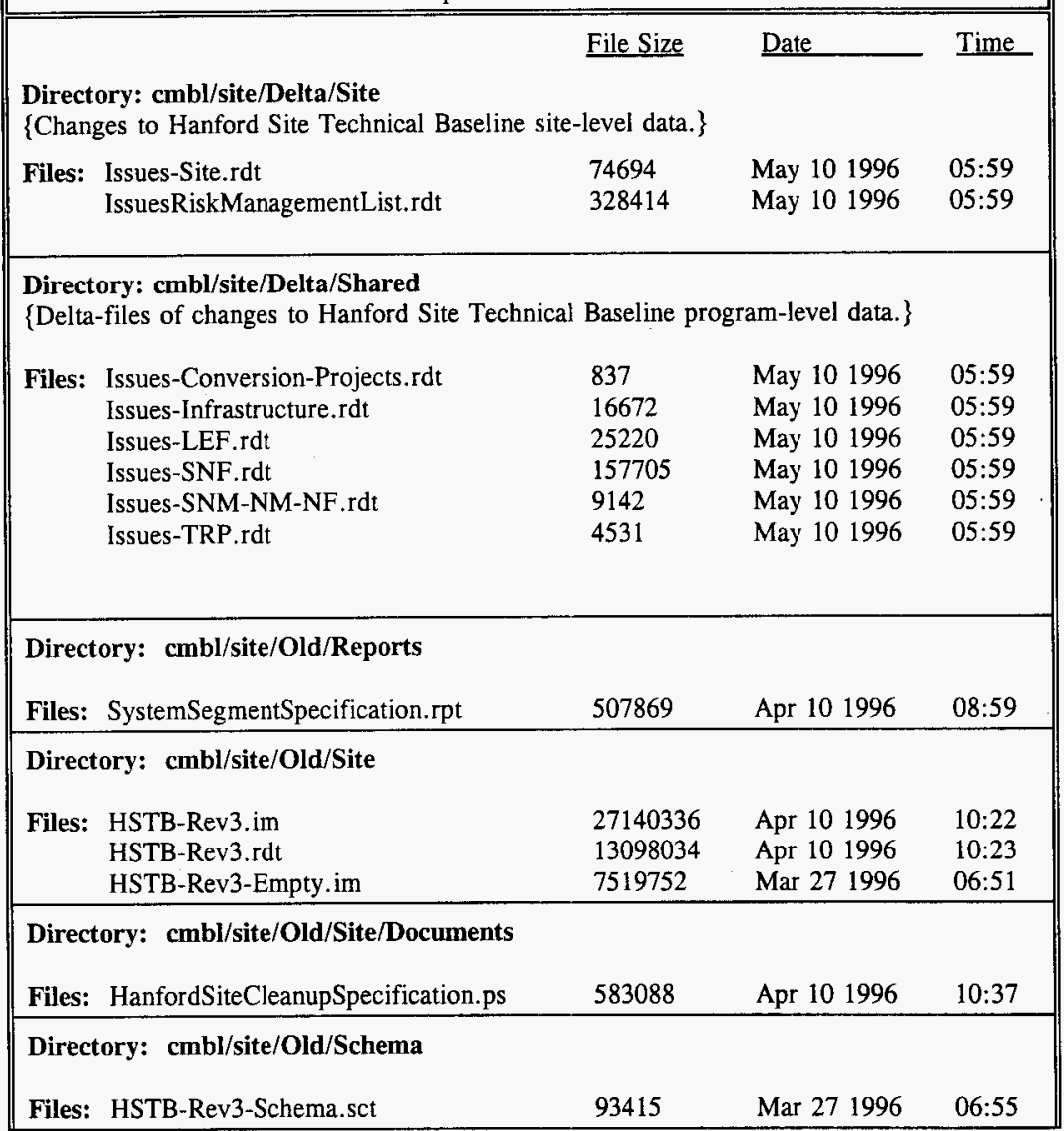




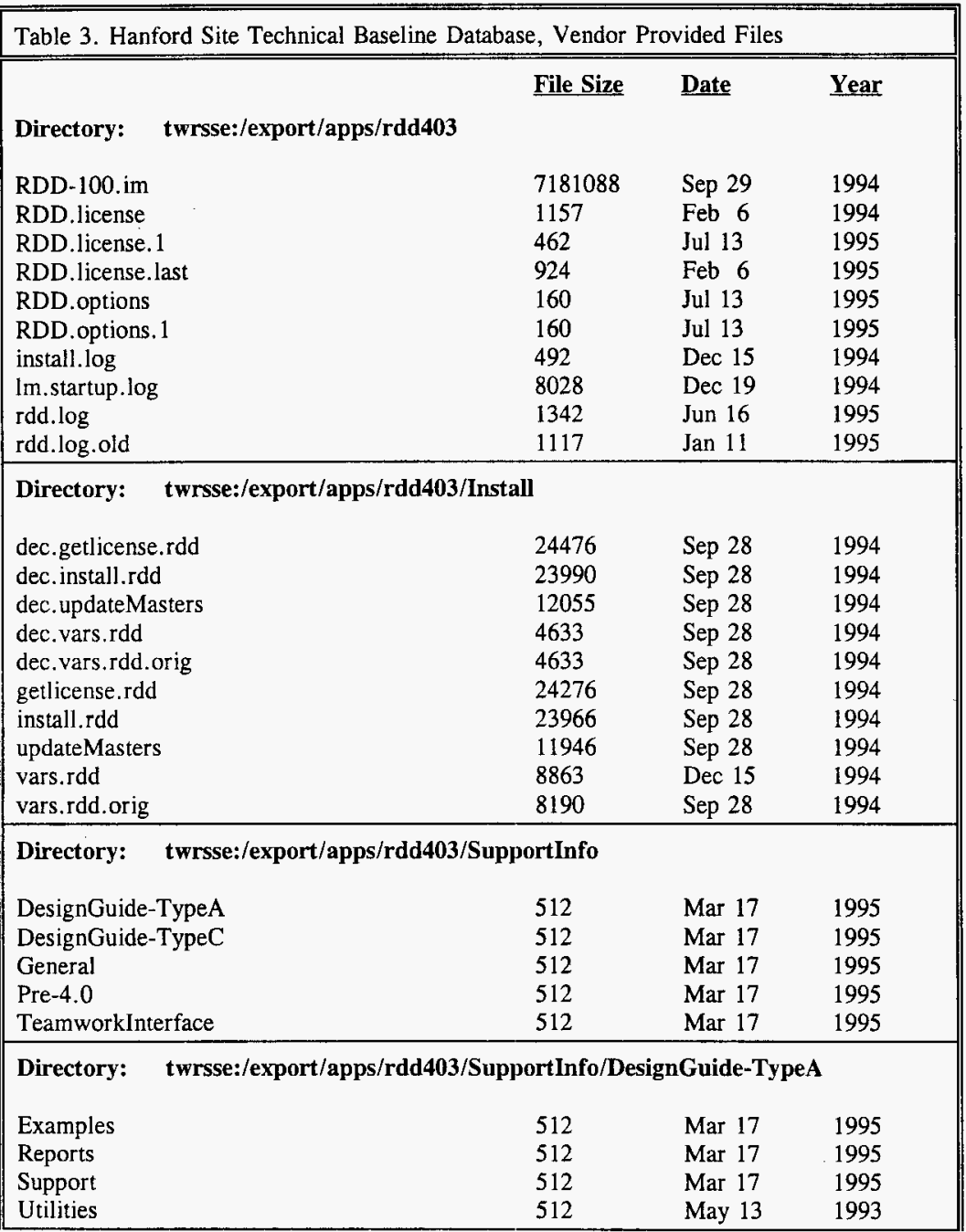


WESTINGHOUSE HANFORD COMPANY

Hanford Site Technical Baseline Database
WHC-SD-GN-CSWD-20012

Rev. 4

Page: 5 of 13

May 10,1996

Table 3. Hanford Site Technical Baseline Database, Vendor Provided Files

\begin{tabular}{|llll} 
& File Size & Date & Year \\
\hline Directory: & twrsse:/export/apps/rdd403/SupportInfo/DesignGuide-TypeA/Examples
\end{tabular}

\begin{tabular}{|c|c|c|c|}
\hline DGA-Model.rdt & 622994 & Apr 20 & 1994 \\
\hline \multicolumn{4}{|c|}{ Directory: twrsse:/export/apps/rdd403/SupportInfo/DesignGuide-TypeA/Repo } \\
\hline DGA-B1Categories.rdt & 3813 & Aug 30 & 1994 \\
\hline DGA-B1Doc.rdt & 17139 & Aug 30 & 1994 \\
\hline DGA-B2Categories.rdt & 3285 & Aug 30 & 1994 \\
\hline DGA-B2Doc.rdt & 14247 & Aug 30 & 1994 \\
\hline DGA-IRSCategories.rdt & 660 & Aug 30 & 1994 \\
\hline DGA-IRSDoc.rdt & 4459 & Aug 30 & 1994 \\
\hline DGA-ProjectUniqueID.rpt & 27106 & Mar 9 & 1994 \\
\hline DGA-Reports.rpt & 1215285 & Sep 6 & 1994 \\
\hline DGA-SRSCategories.rdt & 2676 & Apr 11 & 1994 \\
\hline DGA-SRSDoc.rdt & 13010 & Apr 13 & 1994 \\
\hline DGA-SSDDCategories.rdt & 3264 & $\operatorname{Jan} 25$ & 1994 \\
\hline DGA-SSDDDoc.rdt & 16207 & Aug 30 & 1994 \\
\hline DGA-SSS-ASpecDoc.rdt & 19061 & Aug 30 & 1994 \\
\hline DGA-SSSCategories.rdt & 6018 & Apr 11 & 1994 \\
\hline
\end{tabular}

Directory: twrsse:/export/apps/rdd403/SupportInfo/DesignGuide-TypeA/Support

$\begin{array}{llll}\text { DG-MUMQueries.rqt } & 342583 & \text { Mar 22 } & 1994 \\ \text { DGA-ConsistencyChecks.cct } & 86491 & \text { Mar 31 } & 1994 \\ \text { DGA-MEVTemplates.tpt } & 15873 & \text { Aug 2 } & 1994 \\ \text { DGA-Schema.sct } & 72670 & \text { Sep 8 } & 1994\end{array}$

Directory: twrsse:/export/apps/rdd403/SupportInfo/DesignGuide-TypeC

$\begin{array}{llll}\text { Examples } & 512 & \text { Mar 17 } & 1995 \\ \text { Reports } & 512 & \text { Mar 17 } & 1995 \\ \text { Support } & 512 & \text { Mar 17 } & 1995 \\ \text { Utilities } & 512 & \text { May 13 } & 1993\end{array}$

Directory: twrsse:/export/apps/rdd403/SupportInfo/DesignGuide-TypeC/Examples

$\begin{array}{llll}\text { DGC-Model.rdt } & 905127 & \text { Apr } 20 & 1994\end{array}$ 
Hanford Site Technical Baseline Database

Page:

Table 3. Hanford Site Technical Baseline Database, Vendor Provided Files

\begin{tabular}{|llll||}
\hline & File Size & Date & Year \\
\hline Directory: $\quad$ twrsse:/export/apps/rdd403/SupportInfo/DesignGuide-TypeC/Reports \\
& & & \\
DGC-ProjectUniqueID.rpt & 28929 & May 6 & 1994 \\
DGC-Reports.rpt & 1091441 & Aug 30 & 1994 \\
DGC-SSDDCategories.rdt & 2275 & Mar 15 & 1994 \\
DGC-SSDDDoc.rdt & 12110 & Aug 30 & 1994 \\
DGC-SSS-ASpecDoc.rdt & 19116 & Aug 30 & 1994 \\
DGC-SSSCategories.rdt & 5998 & Mar 15 & 1994 \\
\hline
\end{tabular}


Hanford Site Technical Baseline Database

Page:

Rev. 4

Effective Date:

\begin{tabular}{|c|c|c|c|}
\hline & File Size & Date & Year \\
\hline \multicolumn{4}{|c|}{ twrsse:/export/apps/rdd403/SupportInfo/DesignGuide-TypeC/Support } \\
\hline DG-MUMQueries.rqt & 390092 & May 2 & 1994 \\
\hline DGC-ConsistencyChecks.cet & 143177 & $\operatorname{Dec} 13$ & 1993 \\
\hline DGC-MEVTemplates.tpt & 4611 & Aug 2 & 1994 \\
\hline DGC-Schema.sct & 71515 & Sep 8 & 1994 \\
\hline \multicolumn{4}{|c|}{ Directory: twrsse:/export/apps/rdd403/SupportInfo/General } \\
\hline Examples & 512 & Mar 17 & 1995 \\
\hline Reports & 512 & Mar 17 & 1995 \\
\hline Support & 512 & Mar 17 & 1995 \\
\hline Utilities & 1024 & Mar 17 & 1995 \\
\hline \multicolumn{4}{|c|}{ Directory: twrsse:/export/apps/rdd403/SupportInfo/General/Examples } \\
\hline ATCexample.rdt & 61368 & Dec 2 & 1993 \\
\hline SATCS_Draft_Specs & 2824 & Jun 14 & 1993 \\
\hline Tutorials.rdt & 304626 & Dec 13 & 1993 \\
\hline \multicolumn{4}{|c|}{ Directory: twrsse:/export/apps/rdd403/SupportInfo/General/Reports } \\
\hline ElementDescriptionReport.rpt & 33256 & Mar 29 & 1994 \\
\hline OwnershipReport.rpt & 44542 & May 9 & 1994 \\
\hline SEN-Sections.rdt & 10111 & May 9 & 1994 \\
\hline SchemaDescriptionReport.rpt & 40884 & Apr 13 & 1994 \\
\hline SystemEngineeringNotebook.rpt & 254552 & Sep 27 & 1994 \\
\hline \multicolumn{4}{|c|}{ Directory: twrsse:/export/apps/rdd403/SupportInfo/General/Support } \\
\hline ConsistencyChecks.cet & 87971 & Aug 2 & 1994 \\
\hline MUMQueries.rqt & 283929 & Sep 28 & 1994 \\
\hline StandardTemplates.tpt & 15878 & Aug 16 & 1994 \\
\hline
\end{tabular}


WESTINGHOUSE HANFORD COMPANY

Hanford Site Technical Baseline Database
WHC-SD-GN-CSWD-20012

Rev. 4

Page: 8 of 13

May 10, 1996

\begin{tabular}{|c|c|c|c|}
\hline & File Size & Date & $\underline{\text { Year }}$ \\
\hline \multicolumn{4}{|c|}{ twrsse:/export/apps/rdd403/SupportInfo/General/Utilities } \\
\hline AllocationBuilder.rpt & 49623 & Jun 6 & 1994 \\
\hline ChangeType.rpt & 26863 & Jun 6 & 1994 \\
\hline ComputePerformanceIndexes.rpt & 9480 & May 11 & 1994 \\
\hline DatabaseProfile.rpt & 52066 & Mar 29 & 1994 \\
\hline EraseExtraCRs.rpt & 4127 & Sep 4 & 1993 \\
\hline FindAndReplace.rpt & 29659 & Mar 29 & 1994 \\
\hline FindZeroFooterSize.rpt & 4496 & Mar 25 & 1994 \\
\hline FunctionHierBuilder.rpt & 26831 & Oct 6 & 1993 \\
\hline GhostBuster.rpt & 1973 & Sep 4 & 1993 \\
\hline GhostFinder.rpt & 2168 & Sep 4 & 1993 \\
\hline ItemLevels.rpt & 29226 & Mar 29 & 1994 \\
\hline ListReportMultiColumns.rpt & 3018 & $\operatorname{Mar} 25$ & 1994 \\
\hline ListSelectNodeAnnotations.rpt & 9223 & $\operatorname{Mar} 25$ & 1994 \\
\hline ListSortKeyProblems.rpt & 8862 & Mar 25 & 1994 \\
\hline PrintUserSelectedAttributes.rpt & 20500 & Mar 25 & 1994 \\
\hline ReAuthorElements.rpt & 6956 & Mar 29 & 1994 \\
\hline ReNumberHierarchy.rpt & 20909 & Apr 20 & 1994 \\
\hline RemoveCarriageReturns.rpt & 28304 & Jul 15 & 1994 \\
\hline ReportHierarchy.rqt & 10369 & Mar 25 & 1994 \\
\hline SchemaChanges.rpt & 55832 & Mar 29 & 1994 \\
\hline SourceParser.rpt & 27594 & Aug 16 & 1994 \\
\hline VariantCompare.rpt & 75401 & Aug 29 & 1994 \\
\hline VersionCompare.rpt & 90534 & May 6 & 1994 \\
\hline \multicolumn{4}{|c|}{ Directory: twrsse:/export/apps/rdd403/SupportInfo/Pre-4.0 } \\
\hline Examples & 512 & Aug 5 & 1993 \\
\hline Reports & 512 & Mar 17 & 1995 \\
\hline Support & 512 & Mar 17 & 1995 \\
\hline Utilities & 512 & Mar 17 & 1995 \\
\hline
\end{tabular}




\begin{tabular}{|c|c|c|c|}
\hline & File Size & Date & Year \\
\hline \multicolumn{4}{|c|}{ Directory: twrsse:/export/apps/rdd403/SupportInfo/Pre-4.0/Reports } \\
\hline DIDtemplate.rdt & 78880 & Sep 8 & 1993 \\
\hline IRSConsistencies.rpt & 87821 & Sep 8 & 1993 \\
\hline IRSdocumentElements.rdt & 6031 & Sep 8 & 1993 \\
\hline InterfaceRequirementsSpec.rpt & 123993 & Sep 9 & 1993 \\
\hline RequirementsAllocation.rpt & 94380 & Sep 8 & 1993 \\
\hline RequirementsTraceability.rpt & 69677 & Sep 8 & 1993 \\
\hline SENdocumentElements.rdt & 5032 & Sep 8 & 1993 \\
\hline SSDDConsistencies.rpt & 121910 & Sep 8 & 1993 \\
\hline SSDDdocumentElements.rdt & 19974 & Sep 8 & 1993 \\
\hline SSSConsistencies.rpt & 118905 & Sep 8 & 1993 \\
\hline SSSdocumentElements.rdt & 36290 & Sep 8 & 1993 \\
\hline SystemEngineeringNotebook.rpt & 312040 & Sep 4 & 1993 \\
\hline SystemSegmentDesignDoc.rpt & 214720 & Sep 8 & 1993 \\
\hline SystemSegmentSpecification.rpt & 179412 & Sep 9 & 1993 \\
\hline
\end{tabular}


Table 3. Hanford Site Technical Baseline Database, Vendor Provided Files

File Size Date Year

Directory: twrsse:/export/apps/rdd403/SupportInfo/Pre-4.0/Support

\begin{tabular}{lrrr} 
Compatibility.sct & 537 & Aug 22 & 1993 \\
\hline
\end{tabular}

Directory: twrsse:/export/apps/rdd403/SupportInfo/Pre-4.0/Utilities

$\begin{array}{llll}\text { 3.XReportConverter.rpt } & 10319 & \text { Aug } 25 & 1994\end{array}$

Directory: twrsse:/export/apps/rdd403/SupportInfo/TeamworkInterfaces

$\begin{array}{llll}\text { Reports } & 512 & \text { Mar 17 } & 1995 \\ \text { Utilities } & 512 & \text { Mar 17 } & 1995\end{array}$

Directory: twrsse:/export/apps/rdd403/SupportInfo/TeamworkInterfaces/ Reports

$\begin{array}{llll}\text { DataDictionary.rpt } & 47148 & \text { Jul } 20 & 1993\end{array}$

Directory: twrsse:/export/apps/rdd403/SupportInfo/TeamworkInterfaces/Utilities

$\begin{array}{llll}\text { RDD.Cadre_config } & 348 & \text { Dec } 15 & 1994\end{array}$

RDD.Cadre _menu $\quad 961 \quad$ Dec $15 \quad 1994$

RDD.Cadre prog $\quad 49152 \quad$ Sep 23 1992

Directory: twrsse:/export/apps/bin/rdd403/bin

dec.lm.startup

dec.rdd_4.0.3

Im.startup

mips

rdd403 -> rdd_4.0.3

rdd_4.0.3

$\operatorname{sun} \overline{4}$

sun4-SOL

vars.rdd
8954

15067

8881

512

9

15079

512

512

8863
Sep 28

Sep 28

Sep 28

Mar 17

Mar 17

Sep 28

Mar 17

Jul 7

Dec 15
1994

1994

1994

1995

1995

1994

1995

1995

1994 
Table 3. Hanford Site Technical Baseline Database, Vendor Provided Files

\begin{tabular}{||llll||}
\hline & File Size & Date & Year \\
\hline Directory: & twrsse:/export/apps/rdd403/bin/mips & & \\
ALC_flex & & & \\
RDD-100 & 331776 & Aug 29 & 1994 \\
lmdown & 1075440 & Sep 8 & 1994 \\
lmgrd & 353024 & Sep 2 & 1994 \\
Imbostid & 270336 & Jun 13 & 1994 \\
lmremove & 353024 & Sep 2 & 1994 \\
lmreread & 353024 & Sep 2 & 1994 \\
lmstat & 353024 & Sep 2 & 1994 \\
\hline
\end{tabular}


WESTINGHOUSE HANFORD COMPANY

Hanford Site Technical Baseline Database
WHC-SD-GN-CSWD-20012

Rev. 4

12 of 13

Page:

Effective Date:

\begin{tabular}{|c|c|c|c|}
\hline & File Size & Date & $\underline{\text { Year }}$ \\
\hline \multicolumn{4}{|c|}{ Directory: twrsse:/export/apps/rdd403/bin/sun4 } \\
\hline ALC_flex & 262144 & Aug 29 & 1994 \\
\hline$R D \overline{-1} 100$ & 720896 & Sep 8 & 1994 \\
\hline Imdown & 155648 & May 24 & 1994 \\
\hline lmgrd & 188416 & Jun 9 & 1994 \\
\hline Imhostid & 155648 & May 24 & 1994 \\
\hline Imremove & 155648 & May 24 & 1994 \\
\hline Imreread & 155648 & May 24 & 1994 \\
\hline Imstat & 155648 & May 24 & 1994 \\
\hline \multicolumn{4}{|c|}{ Directory: twrsse:/export/bin/apps/rdd403/sun4-SOL } \\
\hline ALC_flex & 308028 & Aug 29 & 1994 \\
\hline RDD-100 & 964708 & Jul 7 & 1995 \\
\hline RDD-100.orig & 838580 & Sep 8 & 1994 \\
\hline Imdown & 181436 & May 24 & 1994 \\
\hline lmgrd & 229088 & Jun 9 & 1994 \\
\hline lmhostid & 181436 & May 24 & 1994 \\
\hline Imremove & 181436 & May 24 & 1994 \\
\hline Imreread & 181436 & May 24 & 1994 \\
\hline Imstat & 181436 & May 24 & 1994 \\
\hline
\end{tabular}




\begin{tabular}{llr}
\hline WESTINGHOUSE HANFORD COMPANY & & WHC-SD-GN-CSWD-20012 \\
& & Rev. 4 \\
Hanford Site Technical Baseline Database & Page: & 13 of 13 \\
& Effective Date: & May 10, 1996 \\
\hline
\end{tabular}

Table 4. Hanford Site Technical Baseline Database - Platform

\begin{tabular}{||ll|}
\hline Type of computer: & Sun SPARC Station \\
\hline Type of CPU: & SPARCstation 20 \\
\hline Operating system: & SUN OS 4.1.3 or SOLARIS 1.1.b \\
\hline Minimum memory: & 40 Megabytes \\
\hline Minimum hard drive size: & 120 Megabytes \\
\hline Network: & Hanford HLAN \\
\hline Tape Drive: & 8 mm tape drive \\
\hline Other software needs: & Newsprint software program is used for printing. \\
\hline
\end{tabular}

